\title{
Imaging magnetisation dynamics in nano-contact spin-torque vortex oscillators exhibiting gyrotropic mode splitting
}

Paul Steven Keatley ${ }^{1 *}$, Sohrab Redjai Sani ${ }^{2,3,4}$, Gino Hrkac ${ }^{5}$, Seyed Majid Mohseni ${ }^{6}$, Philipp Dürrenfeld ${ }^{7}$, Johan Åkerman ${ }^{2,3,7}$, and Robert J. Hicken ${ }^{1}$

${ }^{1}$ Department of Physics and Astronomy, University of Exeter, Stocker Road, Exeter, EX4 4QL, $U K$.

${ }^{2}$ Materials and Nano Physics, School of ICT, KTH Royal Institute of Technology, Electrum 229, 16460 Kista, Sweden.

${ }^{3}$ NanOsc AB, Electrum 205, 16440 Kista, Sweden.

${ }^{4}$ Department of Physics \& Astronomy, Uppsala University, Box 516, SE-751 20 Uppsala, Sweden.

${ }^{5}$ College of Engineering, Mathematics and Physical Science, University of Exeter, Exeter, EX4 $4 S B, U K$.

${ }^{6}$ Department of Physics, Shahid Beheshti University, G.C., Evin, Tehran 19839, Iran

${ }^{7}$ Physics Department, University of Gothenburg, Fysikgränd 3, 41296 Gothenburg, Sweden

\section{E-mail addresses}

p.s.keatley@exeter.ac.uk (corresponding author)

sohrabrs@kth.se

g.hrkac@exeter.ac.uk

majidm@kth.se

philipp.durrenfeld@physics.gu.se

johan.akerman@physics.gu.se

r.j.hicken@exeter.ac.uk 


\begin{abstract}
Nano-contact spin-torque vortex oscillators (STVOs) are anticipated to find application as nanoscale sources of microwave emission in future technological applications. Presently the output power and phase stability of individual STVOs are not competitive with existing oscillator technologies. Synchronisation of multiple nano-contact STVOs via magnetisation dynamics has been proposed to enhance the microwave emission. The control of device-todevice variations, such as mode splitting of the microwave emission, is essential if multiple STVOs are to be successfully synchronised. In this work a combination of electrical measurements and time-resolved scanning Kerr microscopy (TRSKM) was used to demonstrate how mode splitting in the microwave emission of STVOs was related to the magnetisation dynamics that are generated. The free-running STVO response to a DC current only was used to identify devices and bias magnetic field configurations for which single and multiple modes of microwave emission were observed. Stroboscopic Kerr images were acquired by injecting a small amplitude RF current to phase lock the free-running STVO response. The images showed that the magnetisation dynamics of a multimode device with moderate splitting could be controlled by injecting an RF current so that they exhibit similar spatial character to that of a single mode. Significant splitting was found to result from a complicated equilibrium magnetic state that was observed in Kerr images as irregular spatial characteristics of the magnetisation dynamics. Such dynamics were observed far from the nano-contact and so their presence cannot be detected in electrical measurements. This work demonstrates that TRSKM is a powerful tool for the direct observation of the magnetisation dynamics generated by STVOs that exhibit complicated microwave emission. Characterisation of such dynamics outside the nano-contact perimeter permits a deeper insight into the requirements for optimal phase-locking of multiple STVOs that share common magnetic layers.
\end{abstract}




\section{Introduction}

Spin torque oscillators (STOs)[1],[2],[3],[4] are nanoscale magnetic devices that utilize the transfer of spin angular momentum between constituent magnetic layers [5], [6], [7] to excite magnetisation dynamics. Autonomous (auto) oscillations of an STO are established when a spin transfer torque (STT) counteracts the restoring force or damping of the dynamics. [8] The frequency of such auto-oscillations has been observed through almost three orders of magnitude from $100 \mathrm{MHz}$ to $100 \mathrm{GHz}$ [9]'[10] by varying a DC bias current and an applied magnetic field to control the nature of the magnetisation dynamics. In STOs the DC current is often confined to flow through the device perpendicular to the magnetic layers with high current density $\left(\sim 10^{7}\right.$ to $\left.\sim 10^{8} \mathrm{~A} / \mathrm{cm}^{2}\right)$. This can be achieved by either fabricating a nano-contact (NC) on top of a spin valve multilayer film comprising of a magnetic free and reference layers separated by a non-magnetic metallic spacer[9],[11],[12],[13],[14], or by patterning such a multilayer into a nano-pillar structure[3],[8],[15],[16],[17].

The magnetisation dynamics of nano-pillar STOs are physically confined to the patterned magnetic layers, while in nano-contact STOs the dynamics are free to extend beyond the footprint of the nano-contact and are the subject of this article. These extended dynamics have been used as a mechanism to phase-lock multiple nano-contact STOs that share the same magnetic layers [18],[19],[20],[21],[22],[23]. Such phase-locking is anticipated to overcome the challenges of poor output power and phase stability exhibited by individual devices, which currently prevent their use in technological applications[24],[25].

The spatio-temporal character of the magnetisation dynamics cannot be determined from electrical measurements and is often simulated using micromagnetic models to understand how the dynamics are related to the observed microwave emission. However, a number of optical and x-ray investigations have recently emerged in which the spatial character[26],[27],[28],[29],[30] and temporal evolution [31],[32],[23],[33] of the magnetisation dynamics have been directly observed. Such observations allow the predictions of micromagnetic simulations [20],[34],[35],[36] to be examined [32],[23], while at the same time allowing the conditions required for mutual phase-locking in real devices to be understood. These experimental studies have covered a variety of STO magnetisation dynamics including gigahertz frequency localised[26] and propagating[28] spin waves, solitons[32], and droplets[32], and megahertz frequency vortex gyration[23],[33] as reported in this article.

Auto-oscillations of magnetic vortex gyration can be sustained in the low frequency regime (100 MHz to $1 \mathrm{GHz}$ ) and offer enhanced output power with respect to auto-oscillations 
of magnetisation precession in the same device[37]. The vortex is characterised by in-plane circulating magnetisation surrounding a narrow out-of-plane core of diameter $\sim 10 \mathrm{~nm}$.[38] In so-called spin torque vortex oscillators (STVOs) the magnetisation of the softer free layer is curled into a vortex by the Oersted field (DC Oe-field) associated with a DC current passing through the nano-contact and into the spin valve multilayer with current density $\sim 10^{7}$ to $10^{8} \mathrm{~A} / \mathrm{cm}^{2}$. The same spin polarised current also delivers a STT that excites auto-oscillations of vortex core gyration.[9]'[11] As the core gyrates with frequency $f_{G}$, the reorientation of the free layer magnetisation with respect to that of the reference layer generates a microwave (RF) giant magnetoresistance (GMR) signal with frequency $f_{G}$. The GMR signal corresponds to the relative orientation of the spatially averaged magnetisations of the free and reference layers beneath the nano-contact.[9]

Consistency of nano-contact device-to-device operation is crucial for multiple STVOs to be phase-locked by magnetisation dynamics within shared magnetic layers. Individual device response is understood to be influenced by nanostructural inhomogeneity such as defects, surface roughness and coupling between layers, impurities, and grain orientation in the fixed layer [52]. The local magnetic environment may then vary on length scales that are smaller than the nano-contact diameter, but may influence the gyration of a vortex in a similar way to that of local defects [53]. Unique jumps, changes in gradient, and sometimes splitting of the auto-oscillation frequency are observed as a function of DC current. Such device-todevice variations may also be related to differences in the evolution of the equilibrium magnetic state as the DC current is varied. However, the correlation between these variations in the electrical response and the spatial character of the magnetisation dynamics within the extended magnetic layers remains unreported.

In this work we have used time-resolved scanning Kerr microscopy (TRSKM) to observe the influence of auto-oscillation splitting on the magnetisation dynamics that extend beyond the perimeter of a nano-contact. First we carried out electrical characterisation of the RF emission of two STVOs with nominally identical nano-contacts, each fabricated on its own microscale spin valve mesa. To acquire images of the magnetisation dynamics using TRSKM it is necessary to injection lock the STVO to an RF current. In an STVO exhibiting a single mode of vortex gyration, it has previously been observed that the magnetisation dynamics were defined by localised and far-field spatial characteristics. The localised dynamics were identified as the STT excitation, while the far-field dynamics were shown to be a reorientation of the magnetisation in response to the Oersted field associated with the injected RF current (RF Oe-field). In the same STVO moderate mode splitting was observed for a different bias 
field orientation. However, when the STVO was locked to the injected RF current, the magnetisation dynamics also exhibited well defined localised and far-field dynamics. In contrast, in an STVO exhibiting significant mode splitting the spatial character of the magnetization dynamics was not well defined and revealed evidence of multiple localised excitations within the free layer, even when the device was injection locked. Direct observation of the magnetisation dynamics that correspond to such mode splitting may provide insight into the equilibrium magnetic state and how to achieve controlled synchronisation of multiple STVOs of shared magnetic layers.

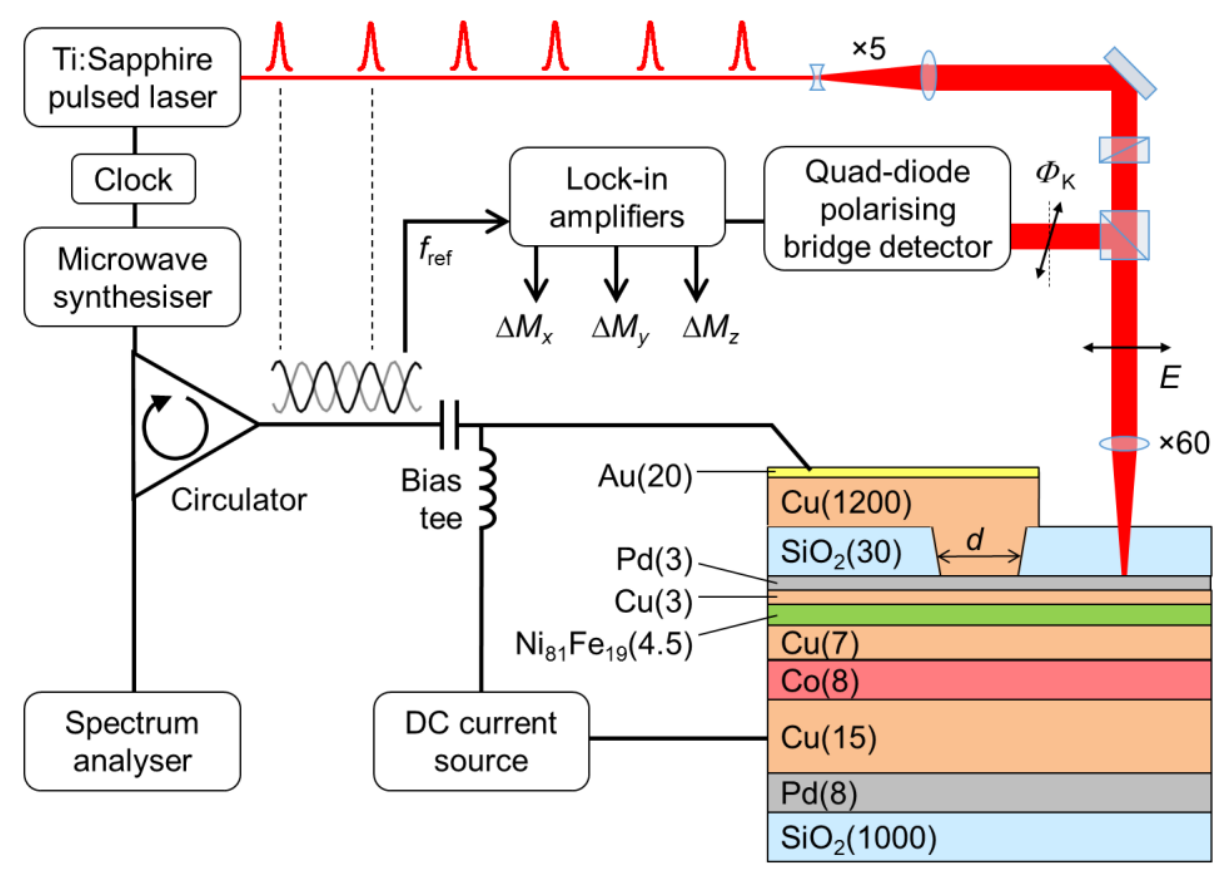

Figure 1. The experimental set-up for combined electrical and optical measurements. Stroboscopic optical measurements were performed by synchronising probe laser pulses to a RF current that was used to injection lock auto-oscillations of the STVO. The RF frequency was set to an integer multiple of the laser pulse repetition rate (here $n=2$ is shown), while phase sensitive detection of the dynamic magnetisation utilised $180^{\circ}$ phase modulation of the RF current at audio frequency $f_{\text {ref. Spatially }}$ dependent MOKE polarisation changes $\Phi_{K}$ of the reflected beam, corresponding to three orthogonal components of the dynamic magnetisation, were recovered simultaneously using a quadrant-diode polarising bridge detector. The incident beam was focused onto the exposed spin valve multilayer structure where the $\mathrm{Cu} / \mathrm{Au}$ of the electrical contact pads had been removed for optical access. The relative layer thickness of the spin valve multilayer are shown to scale (thickness in nm), while the thickness of the $\mathrm{SiO}_{2}$ layers and top Cu layer, and nano-contact diameter $d$, have been reduced for clarity. Adapted from [23] (C) American Physical Society. 


\section{Methods}

Fabrication

In this work two nano-contact STVOs were investigated and are referred to as $\mathrm{NC1}$ and NC2. The two devices had nominally identical nano-contact diameters $d$ of $\sim 250 \mathrm{~nm}$. A schematic cross-section of a nano-contact to a spin-valve multilayer is shown in Figure 1. NC1 and NC2 were fabricated simultaneously on the same four inch silicon wafer. The wafer was covered with a $1 \mu \mathrm{m}$ thick thermally oxidised layer of $\mathrm{SiO}_{2}$. The spin valve film consisted of a $\mathrm{Pd}(8) / \mathrm{Cu}(15) / \mathrm{Co}(8) / \mathrm{Cu}(7) / \mathrm{Ni}_{81} \mathrm{Fe}_{19}(4.5) / \mathrm{Cu}(3) / \mathrm{Pd}(3)$ multilayer (Figure 1, thickness in nanometres) and was deposited using magnetron sputtering with argon plasma and a base pressure below $2 \times 10^{-8}$ Torr. A microscale spin valve mesa with lateral size of $16 \times 8 \mu \mathrm{m}^{2}$ was defined using photolithography and argon dry etching. $\mathrm{A} \mathrm{SiO}_{2}(30)$ layer was then deposited on top of the mesa using plasma enhanced chemical vapour deposition. At the centre of each mesa, the nano-contact was defined in the $\mathrm{SiO}_{2}$ layer using electron-beam lithography and reactive ion etching. A sputtered $\mathrm{Cu}(1200) / \mathrm{Au}(20)$ bilayer was used to form first the $\mathrm{Cu}$ nanocontact to the spin valve multilayer, and then a coplanar waveguide (CPW) using photolithography and lift off techniques, Figure 2(a). The thick $\mathrm{Cu}$ layer provided an effective heat sink for nano-contacts that conduct current densities of up to $\sim 2 \times 10^{8} \mathrm{~A} / \mathrm{cm}^{2}$, while the $\mathrm{Au}$ cap allowed for good electrical contact to the external electronics using Au wire bonds. The CPW was specifically designed to allow optical access to the spin-valve mesa in the vicinity of the nano-contact. The CPW structure and exposed mesa can clearly be seen in the atomic force microscope (AFM) image of Figure 2(b), while the reflectivity image of Figure 2(c) shows how the device appears in the scanning Kerr microscope. The CPW was designed to just overlap the centre of the mesa so that the nano-contact was located at the very edge of the signal pad (Figure 2(b), indicated by arrow ' $N C$ '). In a third, nominally identical device that had expired, AFM images revealed concentric rings of damage extending into the exposed mesa that confirmed that the nano-contact location was at the edge of the signal pad[33]. The $\mathrm{CPW}$ ground planes were electrically contacted to the $\mathrm{Cu} / \mathrm{Pd}$ cap of the mesa using a large $\left(\sim 4 \times 2 \mu \mathrm{m}^{2}\right)$ rectangular vias at each end of the rectangular mesa to promote a symmetric current distribution in the vicinity of the nano-contact. The vias were fabricated using photolithography and the same reactive ion etch used to define the nano-contact in the $\mathrm{SiO}_{2}$. 


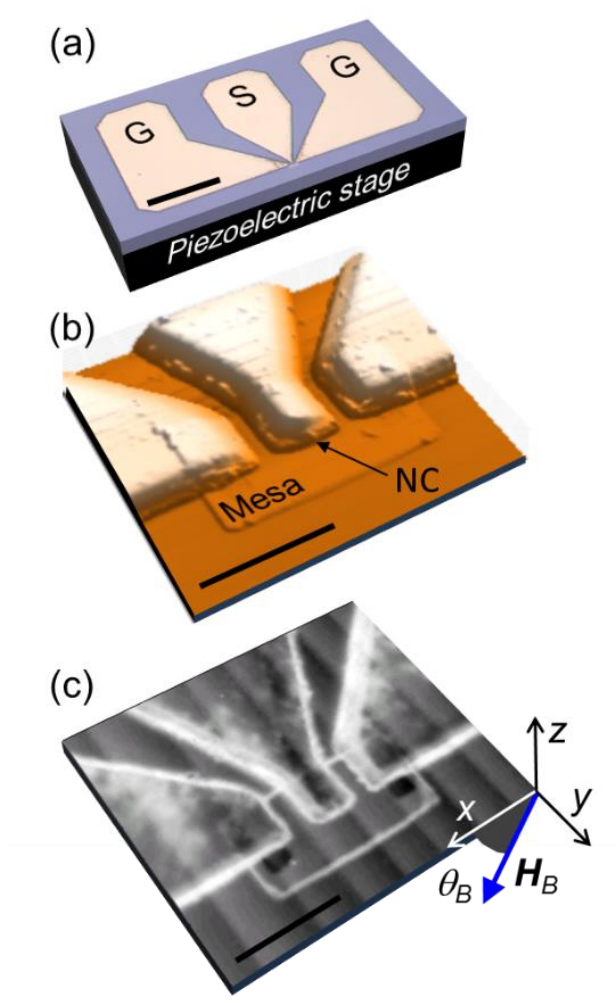

Figure 2. (a) An optical microscope image of the CPW ground-signal-ground (G-S-G) bond pad structure for impedance matched electrical connection to the nano-contact (scale bar $100 \mu \mathrm{m}$ ). (b) An AFM image of the CPW structure in the vicinity of the spin valve mesa (scale bar $10 \mu \mathrm{m}$ ). The nano-contact (NC) was located on the symmetry axis of the CPW structure and at the centre of the mesa (approximate location indicated by arrow). (c) A reflectivity image of the CPW structure and spin valve mesa as they appear in the scanning Kerr microscope with a 400-nm wavelength probe laser (scale bar $10 \mu \mathrm{m}$ ). Partially exposed large rectangular vias (black) can be seen near to the short edges of the rectangular mesa. The overlaid coordinate system is used to define the direction $\theta_{B}$ of the in-plane bias magnetic field $H_{B}$ with respect to the $x$-axis (long edge of the mesa). (a-c) Adapted from [23] and [33] @ American Physical Society.

\section{Electrical characterisation}

The electrical characterisation of the free-running and injection-locked response of the STVOs was carried out in the configuration shown in Figure 1. In measurements of the freerunning response the RF output of the microwave synthesiser was switched off so that only DC current passed through the nano-contact. The DC current $I_{\mathrm{DC}}$ was generated by a precision current source and delivered to the device via a bias tee. Positive DC current was defined as electrons passing from the reference layer to free layer. The GMR signal of the STVO was detected, via the bias tee and a circulator, using a spectrum analyser. At each value of the DC current, the spectrum analyser recorded an average of 5 to 8 power spectra acquired in the range $3 \mathrm{~Hz}$ to $1 \mathrm{GHz}$ using a $100 \mathrm{kHz}$ resolution bandwidth, and $10 \mathrm{~dB}$ of pre-amplification. The 
injection-locked response was measured by using a circulator to simultaneously extract the power spectrum while injecting an RF current $I_{\mathrm{RF}}$ to injection-lock the STVO. The circulator had a minimum isolation of $16 \mathrm{~dB}$ in the range $150 \mathrm{MHz}$ to $250 \mathrm{MHz}$. A vector network analyser was used estimate the insertion loss in the range $30 \mathrm{kHz}$ to $1 \mathrm{GHz}$ from the synthesiser to the nano-contact via the circulator, bias-tee, and connecting RF cables, adapters, planar waveguides, and wire bonds. The microwave synthesiser was phase locked to a $10 \mathrm{MHz}$ output of a low phase noise master clock for TRSKM measurements.

\section{Optical measurements}

Time-resolved Kerr images were acquired using the laser pulses from an ultrafast Ti:Sapphire oscillator that were generated with $\sim 100$ fs duration, $80 \mathrm{MHz}$ repetition rate, and $800 \mathrm{~nm}$ wavelength. To synchronise the laser with the microwave synthesiser, the laser was phase-locked to an $80 \mathrm{MHz}$ output of the master clock by tuning the cavity length. Therefore, to perform stroboscopic TRSKM measurements on STVOs it was necessary for the injected $\mathrm{RF}$ frequency to be an integer multiple of the laser repetition rate $(n \times 80 \mathrm{MHz})$. Unless otherwise stated the frequency of the RF current was $160 \mathrm{MHz}$, or twice the laser repetition rate.

The pulsed laser beam was expanded $\times 5$, linearly polarised, and focused to a diffraction limited spot onto the spin-valve mesa using a $\times 60$ (0.85 numerical aperture) microscope objective lens. The spatial resolution was $\sim 500 \mathrm{~nm}$ at a wavelength of $800 \mathrm{~nm}$. The images were acquired by scanning the mesa beneath the focused laser beam using a piezoelectric stage. Each image was acquired at a fixed phase of the injected RF current. The RF current was phase modulated from 0 to $180^{\circ}$ at $\sim 3 \mathrm{kHz}$ for phase sensitive detection of the corresponding change in the magnetisation, i.e. the dynamic magnetisation. A quadrant photodiode, polarising bridge detector simultaneously detected two in-plane components of the dynamic magnetisation using the longitudinal magneto-optical Kerr effect (MOKE), in addition to the out-of-plane component using the polar MOKE. The Kerr signals corresponding to the vector-components of the dynamic magnetisation were simultaneously recovered at the modulation frequency by three lock-in amplifiers.

\section{Results and discussion}

Typical STVO response

Electrical measurements were performed using negative values of the DC current for which auto-oscillations were observed. Thermal excitation of magnetisation dynamics was 
ruled out by the observed absence of auto-oscillations at positive values of the DC current (not shown). To prepare the devices for TRSKM an in-plane magnetic field of $\sim 20 \mathrm{mT}$ was applied along the intended final direction of the field $\theta_{B}$ and in the absence of a DC current. The resulting saturation of the free and reference layer magnetisations was intended to remove any micromagnetic structure beneath the nano-contact and in its vicinity. The field was then reduced to $5 \mathrm{mT}$ along $\theta_{B}$ for subsequent electrical characterisation and TRSKM measurements. Magneto-resistance and focused Kerr hysteresis loops revealed that the saturation field of the free and reference layers was $\sim 1-2 \mathrm{mT}$ and $\sim 5 \mathrm{mT}$ respectively[23].

After the bias field was set, the DC current was swept from $0 \mathrm{~mA}$ to $-40 \mathrm{~mA}$. In $\mathrm{NC} 1$ a single mode was observed when the bias field was applied along the short edge of the mesa $\left(\left|H_{B}\right|=5 \mathrm{mT}, \theta_{B}=270^{\circ}\right)$, Figure 3(a). Typical abrupt nucleation of the auto-oscillation was observed at $I_{D C}=-30.6 \mathrm{~mA}$. Once nucleated the auto-oscillation was then sustained until a lower cut-off value of $I_{D C}=-13.8 \mathrm{~mA}$, as the DC current was swept from $-40 \mathrm{~mA}$ to $0 \mathrm{~mA}$, Figure 3(b).[6] The in-plane bias field increases the magnitude of the cut-off current due to its competition with the DC Oe-field. Therefore the magnitude of the bias field was chosen to ensure saturation of the free layer magnetisation prior to nucleation, while allowing for a sufficiently low cut off current for the auto-oscillation frequency to pass through at least one integer multiple $n$ of the laser repetition rate. The frequency of NC1 was observed to decrease from approximately $300 \mathrm{MHz}$ to $100 \mathrm{MHz}$ and pass through $160 \mathrm{MHz}(n=2)$ within the operating range of DC current for which a high quality auto-oscillation was observed.

Super-harmonic injection locking (SHIL) of NC1 revealed typical injection locking behaviour of an STVO exhibiting a single mode[33]. In Figure 3(c) the RF frequency was set to $320 \mathrm{MHz}$ so that its remnant spectral signal lay outside of the auto-oscillation frequency range and did not obscure the injection-locked STVO response.[39] When the DC current was again swept from $-40 \mathrm{~mA}$ to $0 \mathrm{~mA}$, frequency pulling and locking of the auto-oscillation were observed between $\sim 22 \mathrm{~mA}$ and $\sim 20 \mathrm{~mA}$. The locking range was observed at a DC current that coincides with the intersection of the injected RF frequency with the second harmonic of the STVO, in accordance with SHIL.[33],[39],[40] The inset of Figure 3(a) shows example power spectra extracted from Figure 3(b) and Figure 3(c) at a DC current of -20.8 mA. Frequency pulling from $162.3 \mathrm{MHz}$ to $160 \mathrm{MHz}$ was observed, in addition to a $1 / 5^{\text {th }}$ reduction of the linewidth (from $5.10 \mathrm{MHz}$ to $980 \mathrm{kHz}$ ) and a $\times 2$ increase in the amplitude (from $8.5 \mathrm{nV} / \mathrm{Hz}^{1 / 2}$ to $15.7 \mathrm{nV} / \mathrm{Hz}^{1 / 2}$ ) with respect to the free-running response to the DC current only. 


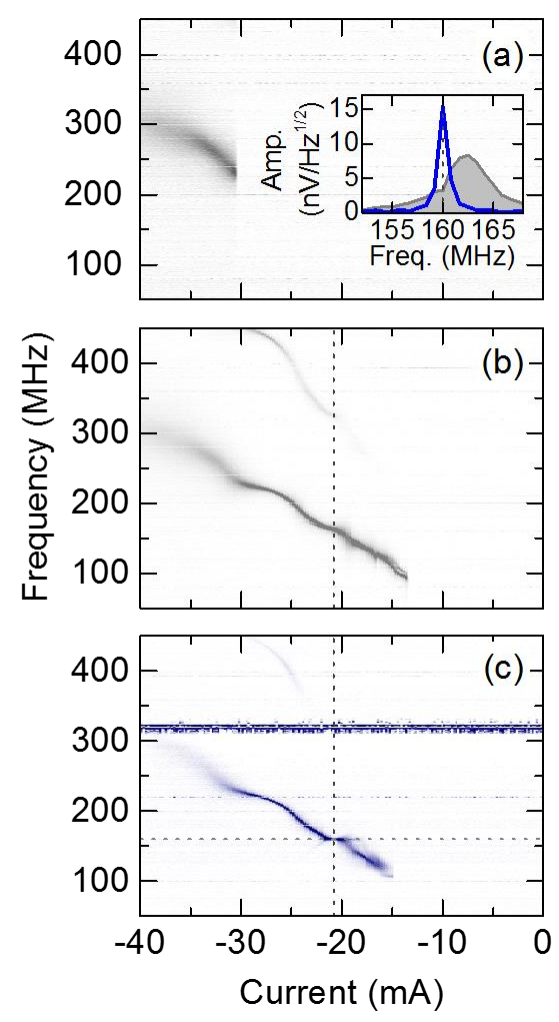

Figure 3. STVO power spectra for NC1 as a function of DC current with an in-plane bias field of $5 \mathrm{mT}$ applied along $\theta_{B}=$ $270^{\circ}$. In (a) the DC current was swept from $0 \mathrm{~mA}$ to $-40 \mathrm{~mA}$. In (b) and (c) the DC current was swept from $-40 \mathrm{~mA}$ to $0 \mathrm{~mA}$ and shows (b) the free-running response to the DC current only and (c) the response to an additional RF current with frequency of $320 \mathrm{MHz}$. In (a) to (c) white and full shading correspond to 0 and $4 \mathrm{nV} / \mathrm{Hz}^{1 / 2}$ respectively. The inset in (a) shows spectra extracted from (b) and (c) at $-20.8 \mathrm{~mA}$ (along vertical dotted lines) showing frequency pulling, enhance amplitude, and reduced linewidth of the injection-locked auto-oscillation. (b) Adapted from [23] @ American Physical Society.

\section{STVO splitting}

In NC1 moderate mode splitting was observed when the bias field was instead applied along the long edge of the mesa $\left(\left|H_{B}\right|=5 \mathrm{mT}, \theta_{B}=0^{\circ}\right)$, Figure 4(a). As the DC current was swept from $-40 \mathrm{~mA}$ to $0 \mathrm{~mA}$ the splitting emerged at $-28.5 \mathrm{~mA}$ and continued until almost the cut-off current at -20 mA. The maximum splitting of $15.8 \mathrm{MHz}$ was observed at $I_{D C} \sim-26 \mathrm{~mA}$. The higher frequency mode exhibited the largest amplitude in the power spectra, Figure 4(c). However, since the GMR signal is generated by the relative orientation of the spatially averaged magnetisations beneath the nano-contact, the spectral amplitude may not be indicative of the amplitude of the magnetisation dynamics within the free layer. For instance, the magnetisation dynamics corresponding to the lower frequency mode may only partially 
enter the perimeter of the nano-contact due to the dominance of the dynamics corresponding to the higher frequency mode beneath the nano-contact.
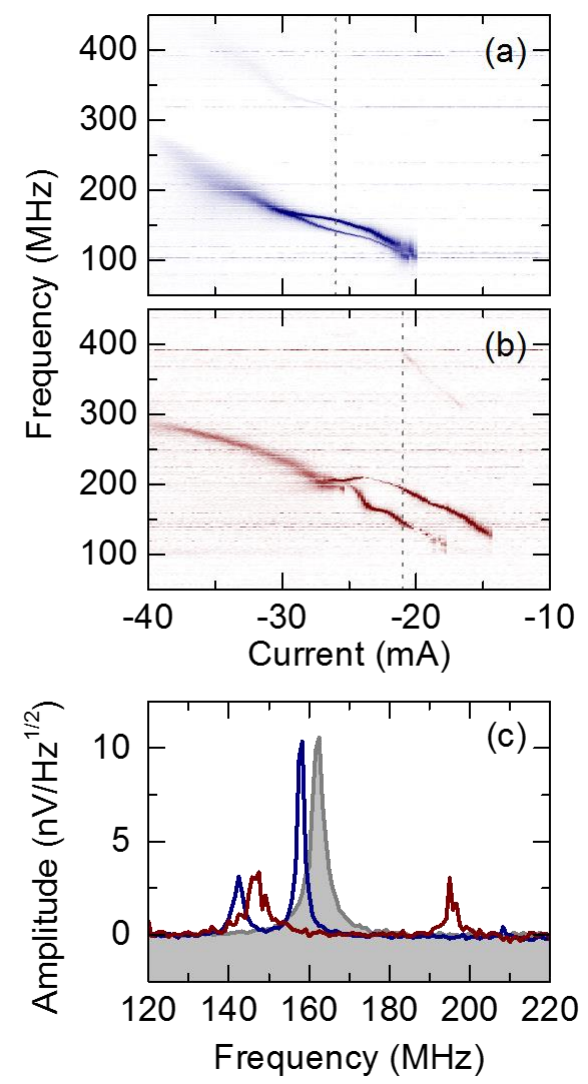

Figure 4. STVO power spectra as a function of DC current with an in-plane bias field of $5 \mathrm{mT}$ applied to (a) NC1 along $\theta_{B}=$ $0^{\circ}$, and (b) NC2 along $\theta_{B}=270^{\circ}$. In (a) and (b) the DC current was swept from $-40 \mathrm{~mA}$ to $0 \mathrm{~mA}$. In (c) spectra corresponding to the current at which maximum splitting is observed in each device (blue and red spectra in (c) correspond to the vertical dotted line at $I_{D C}=-26 \mathrm{~mA}$ in (a) and at $-21 \mathrm{~mA}$ in (b) respectively) are shown overlaid with the spectra extracted from the single mode response of $\mathrm{NC} 1$ (grey spectra in (c) corresponds to the vertical dotted line at $-20.8 \mathrm{~mA}$ in Figure 3(b)). In (a) and (b) white corresponds to $0 \mathrm{nV} / \mathrm{Hz}^{1 / 2}$, while full shading corresponds to 4 and $2 \mathrm{nV} / \mathrm{Hz}^{1 / 2}$ respectively.

In NC2 significant mode splitting was observed when the bias field was again applied along the short edge of the mesa $\left(\left|H_{B}\right|=5 \mathrm{mT}, \theta_{B}=270^{\circ}\right)$, Figure 4(b). As the DC current was swept from $-40 \mathrm{~mA}$ to $0 \mathrm{~mA}$ the splitting emerged from a transition region around $-26 \mathrm{~mA}$ that appeared as a small minimum in the frequency as a function of DC current. The splitting then continued until individual cut-off currents of the higher and lower frequency modes at -14.3 $\mathrm{mA}$ and $-17.5 \mathrm{~mA}$ respectively. The maximum splitting of $49.8 \mathrm{MHz}$ was observed at $I_{D C} \sim-$ $21 \mathrm{~mA}$, while the amplitude of both modes was less than half that of the large amplitude mode of NC1, Figure 4(c). As the DC current was swept gradual transitions of spectral amplitude 
between the two modes occur. This is understood to result from competition between the magnetisation dynamics of each mode beneath the nano-contact as the equilibrium magnetic state evolves in response to the reducing DC Oe-field.

\section{Injection locking}

The free-running STVO behaviour shown in Figure 3(b), Figure 4(a), and Figure 4(b) all show auto-oscillation frequencies that pass through $160 \mathrm{MHz}$. Figure 5 shows each STVO response as the DC current was again swept from $-40 \mathrm{~mA}$ to $0 \mathrm{~mA}$, but with an injected $\mathrm{RF}$ current with frequency of $160 \mathrm{MHz}$. The estimated amplitude of the RF current was $\sim 1.4 \mathrm{~mA}$ $\left(\sim 2.9 \times 10^{6} \mathrm{~A} / \mathrm{cm}^{2}\right)$ after accounting for $\sim 3 \mathrm{~dB}$ of insertion loss. As described earlier, the RF frequency was an integer multiple $(n=2)$ of the laser pulse repetition rate $(80 \mathrm{MHz})$. The remnant signal of the RF current appears as a band around $160 \mathrm{MHz}$ that obscures the STVO response in and around the locking range, Figure 5(a-c).

In NC1 $\left(\theta_{B}=270^{\circ}\right)$ the single mode is slightly pulled towards the RF frequency of $160 \mathrm{MHz}$, locks at $\sim-23.5 \mathrm{~mA}$, and does not reappear, Figure 5(a). This suggests that the autooscillation remains injection-locked until the free running cut-off current as supported by previously reported Kerr images acquired outside of the locking range [23]. An intermodulation mode with frequency $2 f_{R F}-f_{G}$ is observed from around $-30 \mathrm{~mA}$ as the DC current is swept towards the locking range. In $\mathrm{NC} 1\left(\theta_{B}=0^{\circ}\right)$ moderate mode splitting is no longer observed when an RF current is injected, Figure 5(b). Instead injection locking occurs at $\sim-29 \mathrm{~mA}$, just before the splitting emerged in the free-running response. In contrast to the single mode case of $\mathrm{NC} 1$ the auto-oscillation does not exhibit frequency pulling and re-emerges from the locking range at $~-24 \mathrm{~mA}$. While two auto-oscillations are not observed simultaneously outside the locking range, a transition at $-21.5 \mathrm{~mA}$ can be seen. This transition may be ascribed to a mode hop from one auto-oscillation to the other as the equilibrium magnetic state evolves in the reducing DC Oe-field. The injected RF current and associated Oe-field appears to prevent the co-existence of both modes. As for the single auto-oscillation of $\operatorname{NC} 1\left(\theta_{B}=270^{\circ}\right)$ in Figure $5(\mathrm{a})$, intermodulation modes with frequency $2 f_{R F}-f_{G}$ are also observed in the response of $\mathrm{NC} 1\left(\theta_{B}=0^{\circ}\right)$ in Figure 5(b).

In NC2 $\left(\theta_{B}=270^{\circ}\right)$ the DC current at which injection locking takes place is unclear, Figure 5(c). In particular the lower frequency auto-oscillation does not appear as the DC current is swept from $-40 \mathrm{~mA}$ and through the transition region where the splitting emerged in the free running response of Figure 4(b). However, the transition between the single mode and 
the multimode regimes still appears as a minimum in the frequency as a function of DC current at $I_{D C} \sim-26 \mathrm{~mA}$. One tentative explanation is that the lower frequency auto-oscillation is immediately injection-locked once the splitting emerges, while the higher frequency mode remains unlocked, but exhibits substantial frequency pulling. The higher frequency autooscillation continues to exist to $I_{D C} \sim-21.5 \mathrm{~mA}$ at which point it is assumed to be injectionlocked since its free running response retained substantial amplitude to a much lower cut-off current of $-14.3 \mathrm{~mA}$.

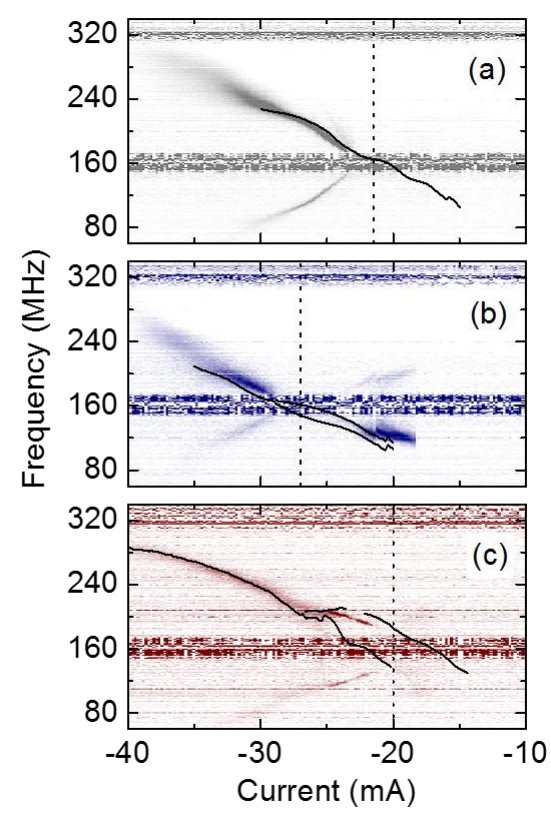

Figure 5. Injection-locked STVO power spectra as a function of DC current with an in-plane bias field of $5 \mathrm{mT}$ applied to device (a) $\mathrm{NC} 1$ along $\theta_{B}=270^{\circ}$, (b) $\mathrm{NC} 1$ along $\theta_{B}=0^{\circ}$, and (c) $\mathrm{NC} 2$ along $\theta_{B}=270^{\circ}$. In (a) to (c) the DC current was swept from $-40 \mathrm{~mA}$ to $0 \mathrm{~mA}$, while the injected RF frequency was $160 \mathrm{MHz}$. In (a), (b), and (c) the free running response of Figures 3(b), 4(a), and 4(b) respectively are shown overlaid as solid black curves, while vertical dotted lines at $-21.5 \mathrm{~mA},-27 \mathrm{~mA}$, and $-20 \mathrm{~mA}$ respectively, indicate the value of $I_{D C}$ at which time-resolved Kerr images were acquired from each device. In (a) to (c) white and full shading correspond to 0 and $1 \mathrm{nV} / \mathrm{Hz}^{1 / 2}$. (a) Adapted from [23] @ American Physical Society.

\section{Time-resolved scanning Kerr microscopy}

Time resolved Kerr images acquired from NC1, with the bias field applied along the short $\left(\theta_{B}=270^{\circ}\right)$ and long $\left(\theta_{B}=0^{\circ}\right)$ edge of the mesa, are shown in Figure 6(a-b) and Figure 6(c-d) respectively. The Kerr images in Figure 6(a-b) were acquired within the locking range at $I_{D C}=-21.5 \mathrm{~mA}$ (vertical dotted line in Figure 3(b)). The images reveal magnetisation dynamics that extend far outside of the nano-contact perimeter and form two well defined regions indicated as regions 1 and 2 in the reflectivity image of Figure 6(b). In region 1 large 
amplitude $\left(\Delta M_{x} \sim 0.5 M_{s}\right)$ localised dynamics are observed in the immediate vicinity of the nano-contact when the phase of the RF current (RF phase) is $0^{\circ}$ (not shown) or $180^{\circ}$, Figure 6(a). Corresponding dynamics in $\Delta M_{y}$ and $\Delta M_{z}$ are also shown in Figure 6(a). Since the RF current is phase modulated and the auto-oscillations are injection-locked, the contrast represents the change in the magnetisation between two dynamic states separated by $180^{\circ}$. Therefore, when the RF phase was shifted from $180^{\circ}$ to $0^{\circ}$ the contrast of Figure 6 (a) was inverted. When the RF phase was swept to $90^{\circ}$ the area of the localised dynamics decreased and almost vanished, while the far-field dynamics of region 2 were observed in $\Delta M_{x}$ images to reach a maximum extending far from the nano-contact, Figure 6(b). A series of time-resolved Kerr images acquired at $15^{\circ}$ phase intervals (not shown) revealed that the far-field dynamics lag the localised dynamics by $90^{\circ}$ [23].

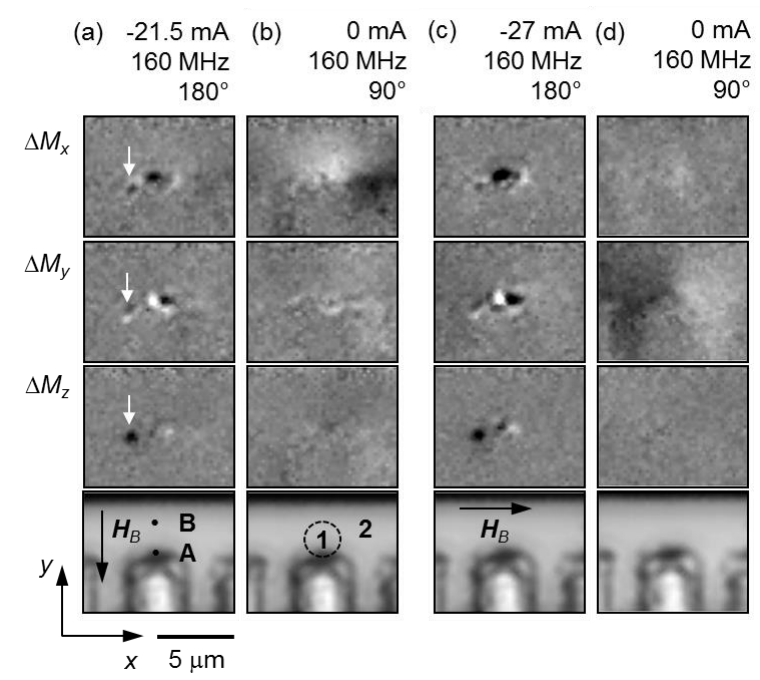

Figure 6. Time-and vector-resolved Kerr images acquired from NC1 with an in-plane bias field of $5 \mathrm{mT}$ applied (a) and (b) along $\theta_{B}=270^{\circ}$, and (c) and (d) along $\theta_{B}=0^{\circ}$ (see bottom panels of (a) and (c)). In (a) to (d) the four panels show (from top to bottom) $\Delta M_{x}, \Delta M_{y}, \Delta M_{z}$ and reflectivity. For images of $\Delta M_{x}$ and $\Delta M_{y}$, black and white represent $+0.5 M_{s}$ and $-0.5 M_{s}$ respectively. The frequency of the injected RF current was $160 \mathrm{MHz}$. In (a) and (c) the RF phase was $180^{\circ}$ and $I_{D C}$ was -21.5 $\mathrm{mA}$ and $-27 \mathrm{~mA}$ respectively. In (b) and (d) the RF phase was 90. In the bottom panel of (b) regions 1 and 2 define the localized and far-field dynamics of (a and c) and (b and d). The white arrows in (a) point to localised dynamics that lie outside of region 1. In (b) and (d) the largest response to the RF Oe-field is observed in images of $\Delta M_{x}$ and $\Delta M y$ respectively due to the different initial torque on the equilibrium magnetisation for bias fields applied along $\theta_{B}=270^{\circ}$ and $0^{\circ}$. (a-b) Adapted from [23] () American Physical Society. 
The injection-locked response of NC1 when $\theta_{B}=0^{\circ}$ (Figure 5(b)) suggested that the moderate mode splitting had been supressed, perhaps in favour of the dominant higher frequency mode of the free-running response. Time-resolved Kerr images support this interpretation. Remarkably, the images in Figure 6(c) reveal localised dynamics with a very similar spatial character to those observed for injection locking of a single mode, Figure 6(a). Crucially, this observation demonstrates that injection locking can control not only the frequency and phase of the auto-oscillation, but also the frequency splitting and spatial character of the localised magnetisation dynamics, even in bias fields with different orientation. At the same time, subtle differences in the spatial character of the localised dynamics can be observed between Figure 6(a) and Figure 6(c). The larger amplitude and area of the localised dynamics in Figure 6(c) are consistent with their acquisition at a larger magnitude of the DC current $\left(\left|I_{D C}\right|=27 \mathrm{~mA}\right)$ than that used to observe dynamics in Figure 6(a) $\left(\left|I_{D C}\right|=21.5 \mathrm{~mA}\right)$.

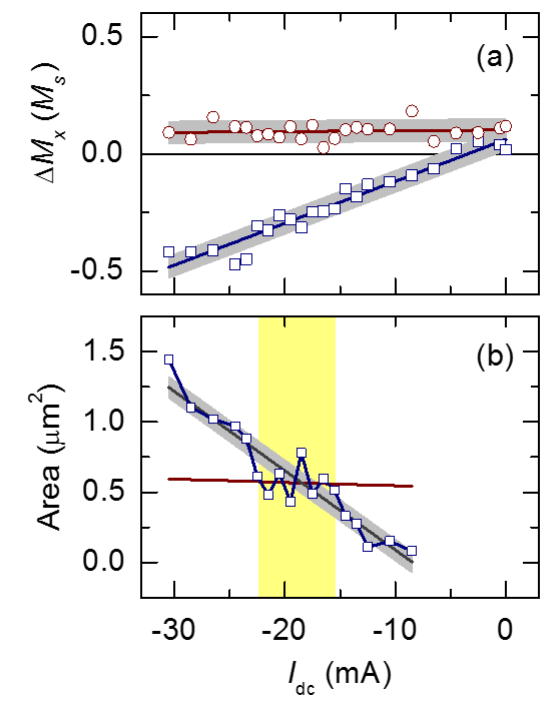

Figure 7. (a) Amplitude of $\Delta M_{x}$ as a function of DC current extracted from Kerr images of NC1 from points A (blue symbols) and B (red symbols) shown in the reflectivity image of Figure 6(a). The images were acquired at a fixed RF phase of $165^{\circ}$ with an in-plane bias field of $5 \mathrm{mT}$ applied along $\theta_{B}=270^{\circ}$. (b) Area of the localised dynamics (A) observed in $\Delta M_{x}$ images as a function of DC current. In (a) and (b), the linear fits are guides to the eye, while the grey shading represents an uncertainty of one standard deviation from the fits calculated from the Kerr signal measured on the CPW signal pad. In (b) the red linear fit is a fitted guide to the eye for the region of reduced gradient. (a-b) Adapted from [23] @ American Physical Society.

The localised dynamics in the immediate vicinity of the nano-contact (Figure 6(a) and (c)) are associated with the gyrotropic auto-oscillation of the vortex core driven by the STT of the DC current.[23],[33] The phase modulation of the RF current leads to the modulation of the vortex core position between two points on its trajectory that are separated by $180^{\circ}$. In 
Kerr images acquired at a particular phase of the RF current, the observed dynamics are the deflection the magnetization between these two dynamic states convoluted with the Gaussian profile of the focused laser beam. The far field dynamics (Figure 6(b) and (d)) are instead a reorientation of the magnetisation in response to the RF Oe-field and can be observed when the DC current is switched off.

To confirm the character of the observed dynamics, the amplitude and area of the localised dynamics generated by $\mathrm{NC} 1$ when $\theta_{B}=270^{\circ}$ (free-running single mode) was previously extracted from $\Delta M_{x}$ images as a function of the DC current[23]. Figure 7(a) reveals a linear decrease in the amplitude of the localised dynamics as the DC current was swept from $-30 \mathrm{~mA}$ to $0 \mathrm{~mA}$. In contrast the amplitude of the far-field dynamics remains constant over the same range of DC current. This confirms that the localised dynamics are driven by STT, while the far-field dynamics are driven by the RF Oe-field and can be observed in the absence of a DC current and associated STT. This interpretation is consistent with the observation of the far-field dynamics in $\Delta M_{x}$ images of Figure 6(b) and $\Delta M_{y}$ images of Figure 6(d) since their equilibrium magnetisations are orthogonal. The torque due to the RF Oe-field therefore acts on orthogonal components of the equilibrium magnetisation. At the same time Figure 7(b) reveals a non-monotonic decrease in the area of the localised dynamics observed in $\Delta M_{x}$ images as the DC current was reduced. This is ascribed to the decrease in the radius of vortex gyration as the STT is reduced. At a DC current of $-23.5 \mathrm{~mA}$ the area of the localised region rapidly decreases at approximately the same DC current as the onset of injection locking in Figure 5(a). The area then appears to stabilise to form a plateau (highlighted by the solid red line in Figure 7(b)) as the DC current is swept towards $-15 \mathrm{~mA}$. This is interpreted as a stabilisation of the radius of gyration when the vortex gyration is phase-locked to the injected RF current.

Kerr images acquired from NC2 when $\theta_{B}=270^{\circ}$ (significant free-running mode splitting) revealed very different dynamics to those of NC1. The spatial character of the dynamics observed at a DC current of $-20 \mathrm{~mA}$ was complicated and did not conform to the localised and far-field regions observed in images acquired from NC1. Instead when the RF phase was $45^{\circ}$ a region of localised dynamics was observed very far from the nano-contact, between the CPW signal pad and right-side ground pad, indicated by arrow in Figure 8(a). When the RF phase was shifted by $180^{\circ}$ to $225^{\circ}$ the localised contrast was inverted. When the RF phase was shifted by a little over $90^{\circ}$ to $150^{\circ}$ the images did not reveal the expected Oefield far-field dynamics that were previously observed in NC1 (Figure 6(b)). Instead a region 
of localised dynamics was observed at a second location far from the nano-contact, indicated by arrow in Figure $8(\mathrm{c})$.

The localised dynamics far from the nano-contact (Figure 8(a) and Figure 8(c)) may be explained by the presence of anti-vortices and perhaps other vortices far from the nano-contact. Micromagnetic simulations reveal that an anti-vortex is formed during the nucleation of the vortex beneath the nano-contact.[35],[36] While the vortex is typically confined to the nanocontact by the restoring effect of the DC Oe-field, the location of the anti-vortex is difficult to control, even in the presence of a bias magnetic field. In numerical models the anti-vortex tends to move towards the edge of the simulated mesh. In real devices it is likely that it will be pinned by magnetic inhomogeneities or trapped in electromagnetic potentials formed by the DC Oe-field. Therefore the localised dynamics of Figure 8(a) and Figure 8(c) may be oscillation of anti-vortices or additional vortices trapped within such potentials. The dynamic feature near to the top-left corner of the CPW signal pad in Figure 6(a) (indicated by arrow) has previously been ascribed to an anti-vortex since it is further from the nano-contact than the spatial extent of the localised dynamics.[23] Remarkably it is also observed in Figure 6(c) after the bias field has been rotated through $90^{\circ}$ suggesting that the pinning is strong. This feature is similar to that observed between the CPW signal pad and the right ground pad in Figure 8(a), while that of Figure 8(c) appears more complicated due to its overlap with the Oe-field dynamics.

To understand if the localised dynamics far from the NC were excited by STT or the RF Oe-field, images of the RF Oe-field excitation (Oe-field images) were acquired. To separate the RF Oe-field excitation from that of the STT, a positive DC current of +20 mA was passed through the nano-contact to attempt to recreate the equilibrium state corresponding to a negative DC current of -20 mA, but with no excitation of auto-oscillations. In Figure 8(b) the Oe-field images show little contrast. Therefore, the localised dynamics of Figure 8(a) may be ascribed to the dynamic interaction of an anti-vortex (or perhaps an additional vortex) with the STT-driven gyration of the vortex beneath the NC. In Figure 8(d) the Oe-field images show some similarities in the spatial character of the dynamics observed at negative DC current, Figure 8(c). However, the large amplitude, highly localised feature of Figure 8(c) (indicted by arrow) is not observed in Figure 8(d) and so again may be ascribed to the dynamic interaction of a vortex or anti-vortex with the STT-driven gyration of the vortex beneath the nano-contact. Such interactions could be mediated by the reorientation of the magnetisation within the free layer as the STT-driven vortex core gyrates beneath the nano-contact. At the same time the 
associated dynamic dipolar fields that are generated by the STT-driven vortex gyration may also excite the localised dynamics of vortices and anti-vortices further from the NC.

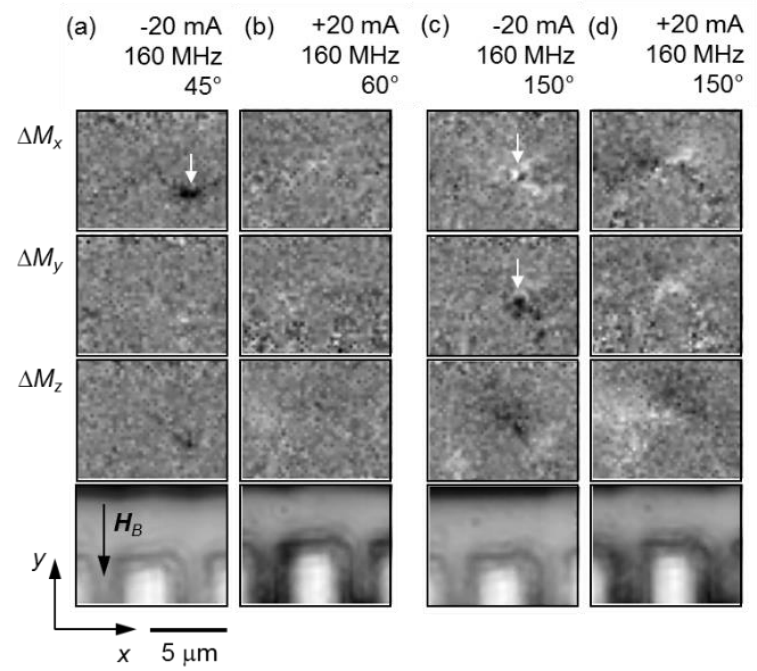

Figure 8. Time-resolved Kerr images acquired from NC2 with an in-plane bias field of $5 \mathrm{mT}$ applied along $\theta_{B}=270^{\circ}$ (see bottom panel of (a)). In (a) to (d) the four panels show (from top to bottom) $\Delta M_{x}, \Delta M_{y}, \Delta M_{z}$, and reflectivity. For images of $\Delta M_{x}$ and $\Delta M_{y}$ black and white represent $+0.25 M_{s}$ and $-0.25 M_{s}$ respectively. The RF frequency and phase were $160 \mathrm{MHz}$ and $160^{\circ}$ respectively. In (a) and (c) $I_{D C}$ was $-20 \mathrm{~mA}$, while in (b) and (d) the DC current was $+20 \mathrm{~mA}$ for which auto oscillations are not observed and the response is driven by the RF Oe-field only. Two regions of localised dynamics, separated in phase by $90^{\circ}$, are indicated in (a) and (c) by a white arrow, while the response to the RF Oe-field in (b) and (c) do not reveal such dynamics.

Localised dynamics far from the nano-contact are not expected to contribute to the power spectra since they are outside of the nano-contact perimeter. However, their dynamic interaction with dynamics beneath the nano-contact may influence the power spectra as the DC current is reduced and the equilibrium magnetisation evolves. The localised dynamics of Figure 8(a) and Figure 8(c) observed far from the nano-contact provide direct insight into the significant mode splitting in the free-running electrical response. Furthermore the difference between the well-defined localised and far-field dynamics of $\mathrm{NC1}$, and the apparent movement of localised dynamics (between Figure 8(a) and Figure 8(c)) far from the nano-contact of NC2, suggests that the equilibrium magnetic state is more complicated in NC2. It is interesting that all of the localised dynamic features of NC2 are observed far from the nano-contact in regions where the DC current density is expected to be high as it flows from the nano-contact to the large rectangular vias at either end of the rectangular mesa. While the DC current density may 
be too weak for direct STT excitation of vortex and anit-vortex dynamics far from the nanocontact, such localised dynamics may instead be driven by the RF Oe-field. However, such localised dynamics were not observed in $\mathrm{NC} 1$ or NC2 in the absence of STT-driven dynamics when $I_{D C}=0 \mathrm{~mA}$ or $+20 \mathrm{~mA}$ (i.e. RF Oe-field excitation only). This supports the interpretation that the dynamic interaction of magnetisation dynamics beneath the nano-contact of NC2 with those far from the nano-contact play a crucial role in the dynamic response of the extended magnetic layers.

Further evidence for a complex equilibrium magnetisation is obtained from the free running response of NC2. Mode competition is observed in which the higher frequency mode appears to vanish as the DC current is swept from $-23.6 \mathrm{~mA}$ and $-22.2 \mathrm{~mA}$, Figure 9(a). Between a DC current of $-25 \mathrm{~mA}$ and $-22.6 \mathrm{~mA}$, the linewidth of the lower frequency mode decreases by a factor $\sim 5$ from 7.2 to $1.5 \mathrm{MHz}$ (Figure 9 (b)), while its amplitude increased by more than one order of magnitude from 0.7 to $9.1 \mathrm{nV} / \mathrm{Hz}^{1 / 2}$ (Figure 9 (c)). Despite the mode competition, the amplitude and linewidth of the modes are comparable to that of $\mathrm{NC1}$ exhibiting a single mode (Figure 9 grey curves).

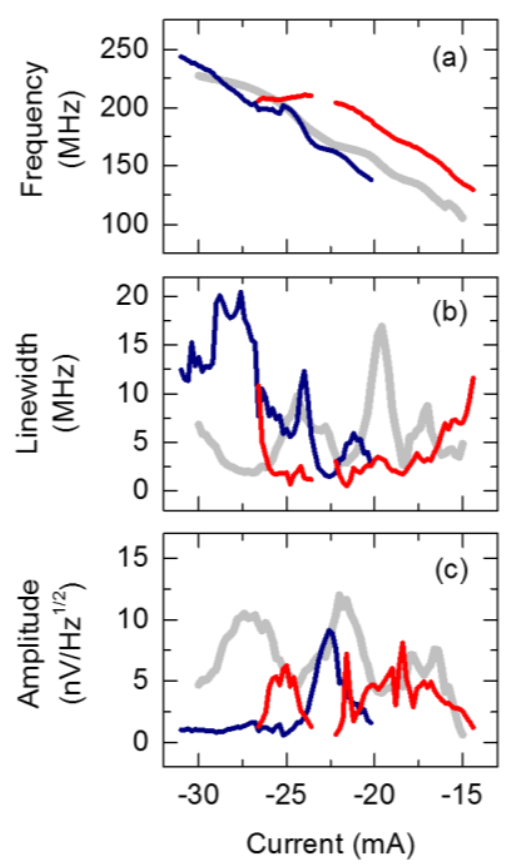

Figure 9. The auto-oscillation frequency (a), linewidth (b), and amplitude (c) extracted from power spectra acquired from devices $\mathrm{NC} 1$ and $\mathrm{NC} 2$ as a function of DC current in a bias field of $5 \mathrm{mT}$ applied along $\theta_{B}=270^{\circ}$. In (a) to (c) the single gyrotropic mode of $\mathrm{NC} 1$ is shown as a grey curve, while the two modes of $\mathrm{NC} 2$ are shown as red and blue curves that correspond respectively to the higher and lower frequency branches after mode splitting takes place. 
The electrical measurements only reveal the spatially averaged response beneath the nano-contact. In contrast the Kerr images reveal that localised dynamics of vortices and/or anti-vortices may exist further from the nano-contact and may not simply vanish as electrical measurements suggest. While the images of Figure 8(a and c) only show the dynamic state at a single DC current, it is plausible that these localised dynamics can migrate within the free layer as the DC current is reduced and the complicated equilibrium magnetic state evolves. Such localised dynamics may interact with the vortex gyration beneath the nano-contact via magnetisation reorientation and/or dynamic dipolar interactions of the shared magnetic layer. As the equilibrium magnetic state evolves, the vortices and/or anti-vortices observed far from the nano-contact may even pass within the immediate vicinity, or perhaps beneath the nanocontact, complicating the power spectra by means of splitting and mode competition summarised in Figure 9.

\section{Summary and conclusion}

In this work we have used a combination of electrical measurements and time-resolved scanning Kerr microscopy to provide insight into the origin of mode splitting in spin-torque vortex oscillators and how moderate splitting may be controlled. STVO device-to-device variation and the variations of an individual device for different bias field configurations was exploited to identify dynamic responses that exhibited a single auto-oscillation of vortex gyration, moderate mode splitting, and significant splitting. Electrical measurements provided some indication that moderate splitting may be controlled to yield an injection locked response similar to that of a single mode, while the injection locked response of a device with significant splitting showed evidence of locked and unlocked modes simultaneously. Time-resolved Kerr images provided direct evidence that for moderate splitting the injection locked response of the localised dynamics was very similar to that of an injection locked single mode, despite differences in the bias field configuration that had a significant influence on the far-field dynamics. In contrast, such well-defined localised vortex gyration and far-field magnetisation reorientation were not formed in a device exhibiting significant splitting. Instead, multiple regions of localised dynamics were observed far from the nano-contact. The localised dynamics were ascribed to the oscillation of vortices and/or antivortices trapped by either magnetic inhomogeneities or electromagnetic potentials far from the nano-contact. It is unlikely that these dynamics can be driven directly by STT, but instead by dynamic interaction via magnetisation reorientation and/or dynamic dipolar fields generated by STT-driven vortex core gyration beneath the nano-contact. Nonetheless, the direct observation of these dynamics 
demonstrate for the first time how complicated the equilibrium magnetic state and corresponding dynamic response of a nano-contact STVO can become, even in the presence of a bias field. The direct observation of the magnetisation dynamics associated with the complicated microwave emission of nano-contact STVOs is crucial to understand how to control device-to-device variations. Dynamics far from the nano-contact are not accessible in electrical measurements, but their observation, characterisation, and control are equally important for the synchronisation of multiple STVOs with common magnetic layers. The combination of such electrical measurements with TRSKM provides a powerful means by which to gain unique insight into the operation of STVOs so that their use as future nanoscale microwave oscillators may be achieved.

\section{Acknowledgments}

The authors gratefully acknowledge the financial support of the Engineering and Physical Sciences Research Council under grants EP/I038470/1 and EP/K008501/1, the Royal Society under grant UF080837, the Swedish Research Council (VR), the Swedish Foundation for Strategic Research (SSF), and the Knut and Alice Wallenberg Foundation (KAW). The authors and co-authors declare that there are no conflicts of interest.

Open access to supporting research data is provided by Open Research Exeter at http://hdl.handle.net/CODE REQUIRED ON ACCEPTANCE.

\section{References}

[1] M. Tsoi, A. G. M. Jansen, J. Bass, W. C. Chiang, M. Seck, V. Tsoi, and P. Wyder, Physical Review Letters 80, 4281 (1998).

[2] M. Tsoi, A. G. M. Jansen, J. Bass, W. C. Chiang, V. Tsoi, and P. Wyder, Nature 406, 46 (2000).

[3] S. I. Kiselev, J. C. Sankey, I. N. Krivorotov, N. C. Emley, R. J. Schoelkopf, R. A. Buhrman, and D. C. Ralph, Nature 425, 380 (2003).

[4] W. H. Rippard, M. R. Pufall, S. Kaka, S. E. Russek, and T. J. Silva, Physical Review Letters 92, 027201 (2004).

[5] J. C. Slonczewski, Journal of Magnetism and Magnetic Materials 159, L1 (1996).

[6] L. Berger, Physical Review B 54, 9353 (1996).

[7] J. C. Slonczewski, Journal of Magnetism and Magnetic Materials 195, L261 (1999).

[8] I. N. Krivorotov, N. C. Emley, J. C. Sankey, S. I. Kiselev, D. C. Ralph, and R. A. Buhrman, Science 307, 228 (2005).

[9] M. R. Pufall, W. H. Rippard, M. L. Schneider, and S. E. Russek, Physical Review B 75, 140404 (2007).

[10] S. Bonetti, P. Muduli, F. Mancoff, and J. Åkerman, Applied Physics Letters 94, 102507 (2009).

[11] Q. Mistral, M. van Kampen, G. Hrkac, J.-V. Kim, T. Devolder, P. Crozat, C. Chappert, L. Lagae, and T. Schrefl, Physical Review Letters 100, 257201 (2008).

[12] T. Devolder et al., Applied Physics Letters 95, 012507 (2009).

[13] M. Kuepferling, C. Serpico, M. Pufall, W. Rippard, T. M. Wallis, A. Imtiaz, P. Krivosik, M. Pasquale, and P. Kabos, Applied Physics Letters 96, 252507 (2010). 
[14] S. R. Sani, J. Persson, S. M. Mohseni, V. Fallahi, and J. Åkerman, Journal of Applied Physics 109, 07 C913 (2011).

[15] D. Houssameddine et al., Nat Mater 6, 447 (2007).

[16] V. S. Pribiag, I. N. Krivorotov, G. D. Fuchs, P. M. Braganca, O. Ozatay, J. C. Sankey, D. C. Ralph, and R. A. Buhrman, Nat Phys 3, 498 (2007).

[17] A. Dussaux et al., Nat Commun 1, 8 (2010).

[18] F. B. Mancoff, N. D. Rizzo, B. N. Engel, and S. Tehrani, Nature 437, 393 (2005).

[19] M. R. Pufall, W. H. Rippard, S. E. Russek, S. Kaka, and J. A. Katine, Physical Review Letters 97, 087206 (2006).

[20] A. Ruotolo et al., Nature Nanotechnology 4, 528 (2009).

[21] S. Sani et al., Nature Communications 4, 2731 (2013).

[22] HoushangA, IacoccaE, DürrenfeldP, S. R. Sani, ÅkermanJ, and R. K. Dumas, Nat Nano 11, 280

(2016).

[23] P. S. Keatley, S. R. Sani, G. Hrkac, S. M. Mohseni, P. Dürrenfeld, T. H. J. Loughran, J. Åkerman, and R. J. Hicken, Physical Review B 94, 060402 (2016).

[24] S. Tamaru, H. Kubota, K. Yakushiji, S. Yuasa, and A. Fukushima, Scientific Reports 5, 18134 (2015).

[25] S. Tamaru, H. Kubota, K. Yakushiji, A. Fukushima, and S. Yuasa, Applied Physics Express 9, 053005 (2016).

[26] V. E. Demidov, S. Urazhdin, and S. O. Demokritov, Nat Mater 9, 984 (2010).

[27] V. E. Demidov, S. Urazhdin, A. Zholud, A. V. Sadovnikov, A. N. Slavin, and S. O. Demokritov, Scientific Reports 5, 8578 (2015).

[28] M. Madami, S. Bonetti, G. Consolo, S. Tacchi, G. Carlotti, G. Gubbiotti, F. B. Mancoff, M. A. Yar, and J. Akerman, Nat Nano 6, 635 (2011).

[29] M. Madami, E. Iacocca, S. Sani, G. Gubbiotti, S. Tacchi, R. K. Dumas, J. Åkerman, and G. Carlotti, Physical Review B 92, 024403 (2015).

[30] D. Backes, F. Macià, S. Bonetti, R. Kukreja, H. Ohldag, and A. D. Kent, Physical Review Letters 115, 127205 (2015).

[31] X. W. Yu et al., Physical Review Letters 106, 167202 (2011).

[32] S. Bonetti et al., Nat Commun 6, 8889 (2015).

[33] P. S. Keatley, S. R. Sani, G. Hrkac, S. M. Mohseni, P. Dürrenfeld, J. Åkerman, and R. J. Hicken, Physical Review B 94, 094404 (2016).

[34] G. Hrkac, T. Schrefl, J. Dean, A. Goncharov, S. Bance, D. Allwood, D. Suess, and J. Fidler, Journal of Applied Physics 105, 083923 (2009).

[35] S. Petit-Watelot et al., Nat Phys 8, 682 (2012).

[36] S. Erokhin and D. Berkov, Physical Review B 89, 144421 (2014).

[37] S. M. Mohseni et al., Science 339, 1295 (2013).

[38] T. Shinjo, T. Okuno, R. Hassdorf, K. Shigeto, and T. Ono, Science 289, 930 (2000).

[39] A. Hamadeh, N. Locatelli, V. V. Naletov, R. Lebrun, G. de Loubens, J. Grollier, O. Klein, and V. Cros, Applied Physics Letters 104, 022408 (2014).

[40] S. Urazhdin, P. Tabor, V. Tiberkevich, and A. Slavin, Physical Review Letters 105, 104101 (2010). 